17 Ueda, K. et al. (1987) J. Biol. Chem. 262 , 17432-17436

18 Goldsmith, M.E. et al. (1993) J. Biol. Chem. 268, 5856-5860

19 Kohno, K et al. (1989) Biochem. Biophys. Res. Commun. 165, 1415-1421

20 Kato, S. et al. (1992) FEBS Lett 308, 175-178

21 Thorgeirsson, S.S. et al. (1991) Pharmacol. Ther. 49, 283-292

22 Descoteaux, S. et al. (1992) Mol. Biochem. Parasitol. 54, 201-212
23 Douch, P.G.C. (1976) Xenobiotica 6. $531-536$

24 Ho, N.F.H. et al. (1992) Mol. Biochem. Parositol. 52, I-14

25 Martin, R.J. et al. (1992) Parasitol. Res. 78 34I-348

26 Callahan, H.L. and Beverley, S.M. (1991) J. Biol. Chem. 266, 18427-18430

27 Rothwell, J.T. and Sangster, N.C. (1993) Int J. Porositol. 23, 573-578

28 Choi, K. et al. (1988) Cell 53, 519-529
29 Loo, T.W. and Clarke, D.M. (1993) J. Biol. Chem. 268, 3143-3149

30 Devine, S.E. et al. (1992) Proc. Natl Acad. Sci USA 89, 4564-4568

3! Gros, P. et al. (1991) Proc. Natl Acad. Sci. USA $88,7289-7293$

Nick Sangster is at the Department of Veterinary Pathology, University of Sydney, New South Wales, 2006, Australia.

\title{
The Role of Chemokines in Schistosoma mansoni Granuloma Formation
}

\author{
N.W. Lukacs, S.L. Kunkel, R.M. Strieter and S.W. Chensue
}

The eradication of parasitic diseases, such as schistosomiasis, has been the focus of investigations worldwide for many decades. However, attempts to control their continual spread have, at best, been met with limited success. In the foce of these results, it is important to attempt to understand and thus to control the pathology of these widespread diseases. In this review, Nicholas Lukacs, Steven Kunkel, Robert Strieter und Stephen Chensue focus on a family of cytokines that play a pertinent role for leukocyte recruitment in chronic inflammation.

Schistosomiasis is a parasitic disease affecting hundreds of millions of people worldwide. The disease is caused by eggs released from helminth worm pairs residing in copula within visceral or pelvic veins. The eggs become lodged in small capillaries of tissues and organs within the host and elicit a granulomatous inflammation. The embryos within the eggs only partially develop. but nevertheless release antigens into the surrounding tissues'. These protein antigens induce a T-cell mediated immunological response ${ }^{2}$ which leads to intense leukocyte recruitment, cellular accumulation and development of $\mathrm{fi}_{-}$ brosing granulomatous reactions around the deposited ovum ${ }^{3}$. Continuous deposition of eggs into the various organs (300 eggs per day per worm pair, lasting for 15-20 years) can lead to severe fibrosis and organ dysfunction'. Not surprisingly, much effort has been applied to elucidating the mechanisms of these destructive responses. Chemotactic cytokines (chemokines) have the ability to induce leukocyte recruitment and, currently, are a major area of investigation for inflammatory research ${ }^{4,5}$. This flurry of interest is due to the therapeutic possibility of controlling leu- kocyte extravasation not only in granulomatous reactions but also in many other acute and chronic inflammatory diseases. This review will examine the role of chemokines and the impact they may have in granulomatous inflammation.

\section{The Role of Chemokines in Inflammation}

The chemokines are divided into two distinct supergene families, termed C-X-C and C-C (based upon amino acid homology and particularly the position of four conserved cysteine amino acids within their primary protein structure $)^{4}$. In general, the C-X-C family, typified by $\mathrm{L}-8$, is largely chemotactic for neutrophils and therefore more closely associated with acute inflammatory reactions, such as endotoxaemia ${ }^{4}$. The C.C family members include macrophage inflammatory protein I (MIP-I) $\alpha / \beta$, monocyte chemoattractant protein I (MCP-I), and RANTES (regulated on activation, normal $T$-cell expressed and secreted). These cytokines are chemotactic for mononuclear cells, monocytes and lymphocytes, and therefore are associated more closely with chronic inflammation, such as schistosome granuloma formation ${ }^{5}$. These criteria are not necessarily absolute as there may be overlapping functional activities.

The recruitment of cells to the area of inflammation is a crucial step in the development of a delayed-type hypersensitivity response. Presumably, the secretion of chemokines contribute to the inflammatory response and are pertinent to the elicitation of particular cell types ${ }^{4.5}$. A chemokine responsible for the recruitment of both lymphocytes and monocytes/macrophages to sites of inflammation is MIP-I6,7. MIP-I $\alpha$ and MIP-I $\beta$ are lipopolysaccharide (LPS)inducible, heparin-binding proteins of $8 \mathrm{kDa}$, and are members of the C-C chemokine supergene family ${ }^{6-8}$. In vivo, the injection of the doublet can induce an acute inflammatory response and prostaglandin-independent fever ${ }^{7,9}$. Interestingly, MIP-I $\alpha$, used individually, has been found to induce the produclion of cylokines, turnor necrusis factor alpha (TNF- $\alpha$ ), IL-I and IL-6 (Ref. I0), thus having the ability to intensify the inflammatory response. This protein is chemotactic for monocytes and T cells, and chemokinetic for neutrophils. MIP-I receptor expression on the various immune cells confirm the latter results, as lymphocytes and monocytes have a high number of specific receptors while neutrophils have a relatively low number" '. Additional studies have demonstrated an interrelationship of MIP-I $\beta$ with the adhesion molecules, CD44 and vascular endothelial cell adhesion molecule I (VCAM-1), as these molecules (in combination with MIP-| $\boldsymbol{\beta}$ ) have been shown to increase CD $8+T$-cell binding to VCAM-I. These findings demonstrate a significant interplay between chemokines and adhesion molecules ${ }^{12}$.

\section{Chemokines in Schistosome Egg Granuloma Formation}

Recent work in our laboratory using Schistosoma mansoni eggs to induce granulomatous responses has demonstrated an important role for MIP-I $\alpha$ in the circumovum response ${ }^{13}$. MIP-| $\alpha$ production during the primary granulomatous response is first apparent as early as Day 4, and continues to increase until peak granuloma formation at Day I6. MIP-I $\alpha$ levels correlate with 
both leukocyte accumulation and the size of the developing granuloma. The production of MIP-l $\alpha$ in secondary granulomas is accelerated, first apparent as early as Day 1, peaking by Day 4, with persistent elevation at Day 8 . These levels again correlate with accelerated accumulation of leukocytes in the developing secondary granuloma. Neutralization of MIP-I $\alpha$, in vivo, during granuloma formation significantly diminishes both the primary and secondary granulomatous responses. Additional studies in our laboratory have suggested that MIP-I $\alpha$ production is regulated by the presence of $\mathrm{CD}^{+}{ }^{-} \mathrm{T}$ cells. These results are consistent with $\mathrm{CD} 4+\mathrm{T}$-cell regulation of granuloma formation'. The predominant cellular source of MIP-| $\alpha$ in the developing granuloma appears to be the activated macrophages that surround the deposited egg. However, fibroblasts isolated and grown in vitro from developing granulornas also have the ability to produce MIP-I $\alpha$ upon stimulation. These latter data are consistent with other studies that have demonstrated stromal cell production of chemokines ${ }^{14-16}$, suggesting a role for these cell types in leukocyte recruitment. In addition, neutrophils have also demonstrated the ability to produce $M I P-1 \alpha$ and may indicate a primary role for these early recruited leukocytes in the initiation of extravasating mononuclear cells to a site of granulomatous inflammation ${ }^{17}$.

Initial reports described MIP-I as a doublet consisting of $\boldsymbol{\alpha}$ and $\boldsymbol{\beta}$ subunits ${ }^{7.8}$. However, subsequent studies demonstrated that the two subunits are products of different genes and can function independently, having different effects on the immune system ${ }^{10}$. MIP-I $\alpha$ is proinflammatory with the ability to activate macrophage function and induce cytokine production. In contrast, MIP-I $\beta$ induces hemopoietic responses and does not have broad inflammatory effects ${ }^{10}$. The production of each of the molecules can counteract the activity of the other, indicating antagonistic outcomes that may have relevance under various conditions in vivo ${ }^{\mathrm{IC}}$. Our studies have demonstrated that MIP-| $\alpha$ has a significant role in granulorna formation and leukocyte accumulation. MIP-I $\beta$ may also contribute to the inflammatory response by inducing hematopoiesis of additional leukocytes to be recruited to the developing lesions.

Other C-C chemokines may also be involved in the development and regulation of the schistosome granuloma. MCP-I is primarily a monocyte chemotactic factor that can be elicited from several cell types including epithelial ${ }^{18}$, endotheliall4, smooth muscle (N.W. Lukacs et al., unpublished) cells, and fibroblasts ${ }^{15,19,20}$. MCP-I can also activate monocytes/macrophages and induce the expression of leukocyte integrins, CDIIb and CDIIC, on the surface of monocytes as well as induce production of IL-I and IL-6 (Ref. 2I). In the schistosome egg-induced lesion, the production of MCP I, similar to MIP-| $\alpha$, correlates with leukocyte accumulation and granuloma size during all phases of the infection (S.W. Chensue et al., unpublished). MCP-I was originally isolated from fibroblasts 18 , and may have a function in mediating fibroblast activation as the granuloma develops. Another C-C family member, RANTES, may also have a role in granuloma formation 5 . This cytokine is chemotactic for monocytes, lymphocytes and eosinophils, and has also been demonstrated to activate and degranulate eosinophils; an event that can cause considerable pathological manifestations ${ }^{22}$. The role of RANTES on eosinophil biology is especially germane to schistosome granuloma formation, since more than $50 \%$ of leukocytes within the lesion are eosinophils.

The induction and regulation of the chemokines (mentioned above) would be pertinent for controlling granuloma formation and size. The induction of the C-C family of chemokines has primarily been described as in response to early response cytokines, $|\mathrm{L}-| \boldsymbol{\beta}$ and TNF $\alpha$. However, the development of the schistosome granuloma has been shown to be regulated by both ThIand Th2-type lymphokines, especially IFN- $\gamma$ and IL-4 which peak during early and peak granuloma formation, respectively 2,3,23,24. Neutralization of IL-4 during the acute secondary granulomatous response diminishes the leukocyte accumulation and correspondingly, lesion size 3,23. This effect may be related to the capacity of IL-4 to induce directly MCP-I production by both endothelial cells ${ }^{19}$ and smooth muscle cell (N.W. Lukacs et al., unpublished). Interestingly, $\mathrm{IL}-10$, initially characterized as a suppressive Th2-type cytokine ${ }^{25}$, can similarly induce MIP-l $\alpha$ production from granuloma fibroblasts ${ }^{26}$. This would suggest a role for mesenchymal cells in the generation of $\mathrm{C}-\mathrm{C}$ chemokines leading to cellular recruitment. These data also suggest that Th2-type lymphokines are able to induce chemokine production from various cell types within the schistosome granuloma. The downregulation of $\mathrm{IL}-4$ and $\mathrm{IL}-10$ at the modulated stage 27 would therefore reduce chemokine production and leukocyte recruitment, thus diminishing granuloma size.

The induction of C-C chemokines during inflammatory schistosome egg granuloma formation is an important event leading to circumovum leukocyte accumulation, sequestration and the destruction of the parasite embryo. Production of C-C family chemokines, $M I P-I \alpha$ and MCP-I, during granuloma formation participates in the recruitment of particular leukocyte subsets to the lesion. However, the overproduction of chemokines can lead to increased cellular activation and possibly severe fibrotic responses, thus damaging the surrounding tissue and eventually causing organ dysfunction. As yet, there is relatively little known about these extremely active cytokines; although the mechanisms of chemokine involvement in granuloma formation are not completely understood, it is likely that they serve an important role in chronic inflammatory events. Future studies should elucidate the specific activating and recruiting roles of the chemokines during chronic inflammatory responses. These studies can be approached utilizing in vivo neutralization of the chemokines with specific antibodies, as well as exogenous administration of recombinant cytokines during homostatic and inflammatory conditions. These studies will demonstrate differential roles for the chemokines for recruitment of specific cell populations to the inflammatory foci and aid in determining mechanisms of cellular localization.

\section{References}

I Boros, D.L. (1989) Clin. Microbiol. Rev, 2, 250-269

2 Grzych, J.M. et al. (1991) J. Immunol. 146, 1322-1329

3 Chensue, S.W. et al. (1992) J. Immunol. 148, 900-906

4 Oppenheim, J.) et al. (1991) Annu. Rev. Immunol 9, 617

5 Schall, T.J. (1991) Cytokine 3, 165-183

6 Davatelis, G. et al. (1990) j. Exp. Med. 167, 1939-1944

7 Wolpe, S.D. et al. (1988) J. Exp. Med. 167. 570-581

8 Wolpe, S.D. and Cerami, A. (1989) FASEB 3, 2565-2573

9 Davatelis, G. et al. (1990) Science 243. 1066-1068

10 Fahey, T.j. et al (1992) J. Immunol. 148, 2764-2769

1 I Wang, J.M. et al. (1993) J. Immunol. 150. 3022-3029

12 Tanaka, Y. et al. (1993) Nature 361, 79-82

13 Lukacs, N.W. et al. (1993) J. Exp. Med. 177, 1551-1559

14 Strieter, RM. et al. (1989) Biochem. Biophys. Res. Commun. 162, 694-700

15 Standiford, T.J. et al. (1993) Chest 103 (Suppl. 2). $1215-1255$

16 Larson, C.G. et al. (1989) Biochern. Biophys. Res. Commun. 160, 1403-1408

17 Kasama, T. et al (1993) f. Exp. Med. 
$178,63-72$

18 Standiford, T.J. et al. (1991) J. Biol. Chem. 266, 9912-9918

19 Rollins, B.J., Morriso, E.D. and Stiles, C.D. (1988) Proc. Natl Acad. Sci. USA 85 , 3738-3742

20 Rollins, B.J. and Pober, J.S. (1991) Am. J. Pathal. 138, 1315-1319

21 Jiang, $Y$. et at. (1992) J. Immunol. 148, 2423-2428

22 Rot, A. et al. (1992) J. Exp. Med. 176,
1489-1495

23 Lukacs, N.W. and Boros, D.L. (1993) Clin. Immunal. Immunopathol. 68, 57-63

24 Lukacs, N.W. and Boros, D.L. (1992) Infect immun. 60, 3209-3216

25 Mosmann, T.R. and Moore, K.W. (1991) in Immunoparasitology Today (Ash, C.P.J. and Gillagher, R.B., eds), pp A49-A58, Elsevier Trends Journals, Cambridge

26 Lukacs, N.W. et ol. (1994) Am. J. Pothol. 144, 7||$-7 \mid 8$
27 Sher, A. et al. (1992) Immunology 147 , $27|3-27| 6$

Nicholos Lukacs, Steven Kunkel and Robert Strieter are at the Departments of Pathology and Intemal Medicine, Division of Critical Care and Pulmonory Medicine, University of Michigan Medical School, and Stephen Chensue is at the Department of Veterans Affairs Medical Center, Ann Arbor, MI 48109-0602, USA.

\title{
MVR-PCR Analysis of Hypervariable DNA Sequence Variation
}

\author{
D.E. Arnot, C. Roper and A.A. Sultan
}

Techniques for accurate marking of infectious microbial agents circulating in populations would be very useful to epidemiologists. In this article, David Amot, Cally Roper and Ali Sultan review recent progress in transfering MVR-PCR DNA fingerprinting techniques from human forensic medicine to parasitology.

A common feature among eukaryotic genomes is the occurrence of mini-satellite repeats comprising short, tandemly reiterated DNA sequences, sometimes referred to as VNTR (variable number of tandem repeats) loci. High levels of inter- and intra-specific variation in the number of tandem repeats can occur at such loci. Where DNA probes exist that hybridize to many dispersed mini-satellite sequences simultaneously, a genome 'fingerprint' can be obtained for use as a taxonomic characteristic. Such techniques have been widely used in parasitology, especially in species where morphological differentiation is difficult (eg. in Leishmania').

\section{The Development of MVR-PCR}

Despite the usefulness of genomic fingerprinting, the method depends on the length variation of target sequences and such variation can be limited. Length variation is also quasi-continuous, making definitive allele identification difficult. This can limit population genetic analysis, since real (but unquantifiable) differences in allelic size usually have to be handled by effectively degrading the information content of the data ('binning') ${ }^{2}$. Minisatellite variant repeat analysis using a specialized polymerase chain reaction
(MVR-PCR, also known as the Jeffreys' method) is a recently developed technique that solves these problems by detecting allelic sequence polymorphism without the effort required by DNA sequencing ${ }^{3}$. The original version of the method exploits the hypervariable minisatellite tandem repeats at a human interstitial locus on chromosome I (the DIS8 locus). There are two types of 29 bp repeat at this locus that differ by a single G-A transition mutation, these are referred to as a-type repeats and $\mathrm{t}$-type repeats. The a-type and t-type repeats show highly diverse interspersion patterns within different alleles. Oligonucleotide primers can be designed that specifically hybridize to each of the variants. Combining such variant repeal specific primers and a primer located at a monomorphic site in the DNA flanking the repeats, it is possible to generate PCR products extending from the flanking primer to each a-type or t-type repeat. Interspersion patterns can then be 'read' from autoradiographs of the separation gel (which have a certain resemblance to supermarket product barcodes) and converted into allelespecific digital codes.

\section{Advantages and Flexibility}

Digitized codes derived from the sequences of individual mini-satellite loci reveal more polymorphism than is detectable by allelic size comparisons and, therefore, more precise identification of individual alleles is achieved. This allows accurate allele-frequency estimation and simplifies standard population-genetic analysis. The technique is relatively simple, rapid and internally well controlled, in that the PCR fragment sizes are predictably spaced in the gel and are thus themselves accurate size markers. This permits accurate comparison of the migration of different lanes, and makes intergel comparisons more reliable. Digital codes are also easier to compile and compare using computers. The main obstacle to the adoption of a MVR-PCR system is that of finding suitable MVR-PCR loci. Repeated DNA sequences flanked by conserved regions exist in most eukaryotes but few have been conveniently characterized. An exception is Plasmodium falciparum, which has many genes containing tandemly repeated DNA encoding conserved, repetitive amino acid sequences. We have used these features of the $P$. falciparum circumsporozoite (CS) gene to develop an MVR-PCR assay that can reveal very high levels of DNA sequence polymorphism at this locus ${ }^{4}$. Box I illustrates the principles of the technique as applied to an ideal target locus in a haploid organism such as $P$. folciporum. MVR-PCR methodology must be adapted according to the ploidy of the organism, the size of the tandemly repeated unit and the extent of conservation of the flanking sequences. The $P$. falciporum system is adapted to the CS gene tandemrepeat unit of $12 \mathrm{bp}$ and, since primers have to be around $20 \mathrm{bp}$, our CS gene variant-specific primers overlap two units. In CS genes inter-repeat variation creates 'null' positions that do not crosshybridize with either primer and these positions are interspersed in the repeat array and contribute to allelic polymorphism. The results of MVR-PCR reactions on DNA extracted from blood 